\title{
Transient electrophoresis of dielectric spheres
}

\author{
Huan J. Keh*, You C. Huang \\ Department of Chemical Engineering, National Taiwan University, Taipei 10617, Taiwan, Republic of China
}

Received 3 February 2005; accepted 28 April 2005

Available online 28 June 2005

\begin{abstract}
The dynamic electrophoretic response of a spherical dielectric particle suspended in an electrolyte solution to a step change in the applied electrics field is analytically studied. The electrical double layer surrounding the particle may have either a small but finite thickness or a very large thickness relative to the particle radius. For the case of electrophoresis of a particle with a thin double layer, the local electroosmotic velocity at the outer edge of the double layer evolving with time after the external field is imposed is used as an apparent slip boundary condition at the particle surface so that the unsteady equation of motion for the fluid flow outside the double layer is solved. Closed-form formulas for the transient electrophoretic mobility of the particle are derived as functions of relevant parameters. The results demonstrate that, when the double layer surrounding the particle is relatively thin, the normalized electrophoretic mobility at a given dimensionless time decreases monotonically with a decrease in the parameter $\kappa a$, where $\kappa^{-1}$ is the Debye screening length and $a$ is the particle radius. When the double layer of the particle is relatively thick, the particle mobility can have magnitudes comparable to those for a particle with a thin double layer in the initial stage, but will become much smaller afterward. In general, the effect of the relaxation time for transient electrophoresis is negligible, regardless of the value of $\kappa a$.
\end{abstract}

(c) 2005 Elsevier Inc. All rights reserved.

Keywords: Electrophoresis; Electroosmosis; Transient response; Step-function electric field

\section{Introduction}

Electrophoresis is a well-known technique that can be used in different fields such as clinical and biological fields to separate and identify compounds such as amino acids and proteins. Although the basic relationships involved in electrophoretic phenomena were derived mainly for the steady state [1-13], the transient behavior of these phenomena is perhaps as important as their steady-state behavior insofar as an evaluation of their usefulness or an efficient design of the relevant equipment is concerned. When a colloidal particle moves through a constant but nonuniform electric field, the field measured in the frame of the particle is unsteady. In several applications, the use of alternating electric fields for measurement of the electrophoretic mobility of suspended particles has been proposed [14,15]. Knowledge of the dy-

\footnotetext{
* Corresponding author.

E-mail address: huan@ntu.edu.tw (H.J. Keh).
}

namic response of such particles to a time-variant electric field can be used to develop new separation technologies or to interpret experimental observations.

Applying the ad hoc assumption that the fluid in the electrical double layer surrounding the particle attains its full electroosmotic velocity instantaneously when the constant electric field is imposed, Morrison found exact solutions for the transient electrophoresis of a dielectric sphere [16] and of an arbitrarily oriented long cylinder [17] for the limit of vanishingly thin double layers $\left(\kappa a \rightarrow \infty\right.$, where $\kappa^{-1}$ is the Debye screening length and $a$ is the particle radius). Later, Ivory [18] made a correction to Morrison's solution for the electrophoresis of a sphere by applying the interfacial boundary condition derived from the integral form of the momentum equation governing the fluid motion in the double layer, but this model failed to predict the transient response of the particle as a function of the parameter $\kappa a$.

In some practical applications of electrophoresis involving small particles and dilute electrolyte solutions, the condition $\kappa a \rightarrow \infty$ is no longer satisfied and the dependence 
of the dynamic response of an electrophoretic particle on $\kappa a$ must be taken into account. In this paper we present an analysis of the transient electrophoresis of a dielectric sphere due to a sudden application of a constant electric field for two cases: a particle with a thin but finite double layer $(\kappa a \gg 1)$ and a particle with a thick double layer $(\kappa a \rightarrow 0)$. The zeta potential (or surface charge density) of the particle is assumed to be uniform, but, for the case of a thin but finite double layer, the dynamic response of the electroosmotic flow at the particle surface is incorporated. The transient responses of the electrophoretic mobility of the particle to a step change in the applied electric field as functions of the relevant parameters such as $\kappa a$ are obtained in closed forms.

\section{Transient electroosmotic flow parallel to a dielectric plane}

We first consider the transient laminar flow of an electrolyte solution within the electric double layer adjacent to a dielectric plane wall under the influence of a constant electric field $E$ applied tangential to the wall starting at the time $t=0$. The electroosmotic velocity at the outer edge of the double layer will serve as a boundary condition for the formulation of the transient electrophoresis of a spherical particle with a thin double layer in Section 3. The discrete nature of the surface charges, which are uniformly distributed over the wall, is neglected, so that the space charge density and equilibrium electric potential are functions of the normal position only. Gravitational effects are ignored. The fluid velocity in the direction of the electric field at lateral position $y$ and time $t, u(y, t)$, which satisfies the equation of continuity for an incompressible fluid, is governed by the Navier-Stokes equation modified with the electrostatic effect [19],

$\rho \frac{\partial u}{\partial t}=\eta \frac{\partial^{2} u}{\partial y^{2}}+\rho_{\mathrm{e}} E$,

where $\rho$ and $\eta$ are the density and viscosity, respectively, of the fluid. The net space charge density $\rho_{\mathrm{e}}(y)$ is related to the equilibrium electric potential distribution $\psi(y)$ by Poisson equation $[3,19]$

$\frac{d^{2} \psi}{d y^{2}}=-\frac{4 \pi}{\varepsilon} \rho_{\mathrm{e}}$,

based on the assumption that the ionic diffusion time scale across the double layer is much shorter than the viscous time scale such that the lateral potential profile is not affected by the tangentially applied electric field. In this equation $\varepsilon=$ $4 \pi \varepsilon_{0} \varepsilon_{\mathrm{r}}$, where $\varepsilon_{\mathrm{r}}$ is the relative permittivity of the electrolyte solution and $\varepsilon_{0}$ is the permittivity of a vacuum. Note that the contributions from the pressure gradient and the gradient of the equilibrium potential cancel out in the Navier-Stokes equation.
The initial and boundary conditions for $u$ are

$$
\begin{array}{ll}
t=0: \quad u=0, \\
y=0: \quad \psi=\zeta \quad \text { and } \quad u=0, \\
y=\delta: & \frac{d \psi}{d y}=0 \quad \text { and } \quad \frac{\partial u}{\partial y}=0,
\end{array}
$$

where $\zeta$ is the zeta potential or surface potential at the wall (shear plane) and $y=\delta$ represents the outer edge of the electric double layer. Evidently, $\delta$ can be taken equal to $b \kappa^{-1}$, where $\kappa^{-1}$ is the Debye length and $b$ is a positive number about 3 to 5 .

Since the system will attain a steady state as $t \rightarrow \infty$, a solution to Eq. (1) of the following form ought to be sought:

$u(y, t)=u_{\infty}(y)+u_{\mathrm{t}}(y, t)$.

The steady-state limiting solution $u_{\infty}(y)$ is obtained from Eqs. (1) and (2) by setting $\partial u / \partial t=0$, for which the result satisfying boundary conditions (4) and (5) is the Helmholtz expression [1],

$u_{\infty}=-\frac{\varepsilon E}{4 \pi \eta}[\zeta-\psi(y)]$.

Clearly, $\psi(\delta)=0$ and $u_{\infty}(\delta)=-\varepsilon \zeta E / 4 \pi \eta$. Inserting Eq. (6) into Eqs. (1)-(5) after the substitution of Eq. (7), one obtains the governing equation as well as the initial and boundary conditions for the transient velocity function $u_{\mathrm{t}}(y, t)$,

$\frac{\partial u_{\mathrm{t}}}{\partial t}=v \frac{\partial^{2} u_{\mathrm{t}}}{\partial y^{2}}$

and

$t=0: \quad u_{\mathrm{t}}=-u_{\infty}(y)$,

$y=0: \quad u_{\mathrm{t}}=0$

$y=\delta: \quad \frac{\partial u_{\mathrm{t}}}{\partial y}=0$,

where $v=\eta / \rho$ is the kinematic viscosity of the fluid. These equations can be solved by the classical method of separation of variables, and the solution is

$$
\begin{aligned}
u_{\mathrm{t}}= & -\sum_{n=1}^{\infty}\left[\frac{2}{\delta} \int_{0}^{\delta} u_{\infty}(y) \sin \left(D_{n} y\right) d y\right] \\
& \times \sin \left(D_{n} y\right) \exp \left(-D_{n}^{2} v t\right),
\end{aligned}
$$

where

$D_{n}=\frac{(2 n-1) \pi}{2 \delta}$.

The combination of Eqs. (7) and (12) according to Eq. (6) gives the fluid velocity profile $u(y, t)$ along the plane wall. As expected, the solution of this transient flow problem comes out in the form of infinite series. This series converges rapidly for large dimensionless times $v t / \delta^{2}$.

The fluid velocity at a large distance from the plane wall (with $\kappa y \geqslant \kappa \delta=b$ ) caused by the suddenly applied electric 
field can be evaluated from the expression for $u$ in Eq. (6), noting that $\psi(y) \rightarrow 0$ far from the wall. The result of this time-dependent electroosmotic velocity, $U_{\delta}(t)$, is

$U_{\delta}=-\frac{\varepsilon \zeta E}{4 \pi \eta}\left[1+\sum_{n=1}^{\infty} C_{n} \exp \left(-D_{n}^{2} \nu t\right)\right]$,

where

$C_{n}=\frac{4(-1)^{n}}{(2 n-1) \pi}$.

Taking $\delta$ as the half thickness of a capillary slit, Eqs. (12) and (14) can also represent the corresponding electroosmotic velocity of the fluid within the slit [19]. Note that, in Eq. (14), the unsteady part retains its dependence on the double-layer thickness.

\section{Transient electrophoresis of a sphere with a thin double layer}

In this section, the transient electrophoretic motion of a dielectric sphere of radius $a$ surrounded by a thin but finite electric double layer in an electrolyte solution (viz. $\kappa a \gg 1$ ) is analyzed. The spherical coordinates $(r, \theta, \phi)$ with its origin at the particle center are used. At the time $t=0$, the uniform electric field is imposed in the positive $z$ direction and maintains a constant strength $E_{\infty}$ throughout the system. Because the Reynolds number of electrokinetic flows is small, the motion of the fluid outside the thin double layer, which is electrically neutral, is governed by the unsteady equation of motion for viscous axisymmetric creeping flows,

$E_{\mathrm{S}}^{2}\left(E_{\mathrm{S}}^{2}-\frac{1}{v} \frac{\partial}{\partial t}\right) \Psi=0$

in which the Stokes stream function $\Psi$ is related to the velocity components by

$v_{r}=-\frac{1}{r^{2} \sin \theta} \frac{\partial \Psi}{\partial \theta}$

$v_{\theta}=\frac{1}{r \sin \theta} \frac{\partial \Psi}{\partial r}$,

and the Stokes operator $E_{\mathrm{s}}^{2}$ has the form

$E_{\mathrm{S}}^{2}=\frac{\partial^{2}}{\partial r^{2}}+\frac{\sin \theta}{r^{2}} \frac{\partial}{\partial \theta}\left(\frac{1}{\sin \theta} \frac{\partial}{\partial \theta}\right)$.

It can be shown that the local tangential electric field at the surface of the dielectric sphere caused by the imposed electric field equals $(-3 / 2) E_{\infty} \sin \theta[1,13]$. This local electric field acting on the diffuse ions within the thin double layer at the particle surface produces a relative tangential fluid velocity at the outer edge of the double layer as given by Eq. (14). Thus, the initial and boundary conditions for the flow field around the particle are

$t=0: \quad v_{r}=v_{\theta}=0$,

$$
\begin{aligned}
r=a^{+}: \quad v_{r}= & U \cos \theta, \\
v_{\theta}= & -U \sin \theta+\frac{\varepsilon \zeta}{4 \pi \eta}\left[1+\sum_{n=1}^{\infty} C_{n} \exp \left(-D_{n}^{2} \nu t\right)\right] \\
& \times \frac{3}{2} E_{\infty} \sin \theta,
\end{aligned}
$$

$r \rightarrow \infty: \quad v_{r}=v_{\theta}=0$,

where $r=a^{+}$represents the outer edge of the thin double layer, $U(t)$ is the time-dependent electrophoretic velocity of the particle to be determined, which can be expressed as $U=U_{\infty} \mu(t)$ with the steady particle velocity given by the Smoluchowski equation [1],

$U_{\infty} \equiv \frac{\varepsilon \zeta E_{\infty}}{4 \pi \eta}$,

and $\zeta$ is the zeta potential of the particle. Clearly, $\mu(0)=0$ and $\mu(\infty)=1$.

The Laplace transform, which is defined by an overbar for a function of time $f(t)$ as

$\bar{f}(s)=\int_{0}^{\infty} f(t) \exp (-s t) d t$

and

$f(t)=\frac{1}{2 \pi i} \int_{\gamma-i \infty}^{\gamma+i \infty} \bar{f}(s) \exp (s t) d s$,

with $i=\sqrt{-1}$, will be used to solve for the flow field and particle velocity. Then, the transform of the general solution of Eqs. (16) and (21) can be expressed as [16]

$$
\begin{aligned}
\bar{\Psi}=\frac{3}{2} U_{\infty} a \sqrt{\frac{v}{s}} & {\left[A \sqrt{\frac{v}{s}} \frac{1}{r}+B\left(1+\sqrt{\frac{v}{s}} \frac{1}{r}\right)\right.} \\
& \left.\times \exp \left(-\sqrt{\frac{s}{v}} r\right)\right] \sin ^{2} \theta,
\end{aligned}
$$

where the coefficients $A$ and $B$ are to be determined from the boundary conditions in Eq. (20), with the result

$$
\begin{aligned}
A= & \left(1+a \sqrt{\frac{s}{v}}\right)\left(\frac{1}{s}+\sum_{n=1}^{\infty} \frac{C_{n}}{D_{n}^{2} v+s}\right) \\
& -\left(1+a \sqrt{\frac{s}{v}}+\frac{a^{2}}{3} \frac{s}{v}\right) \bar{\mu}(s), \\
B= & {\left[\bar{\mu}(s)-\frac{1}{s}-\sum_{n=1}^{\infty} \frac{C_{n}}{D_{n}^{2} v+s}\right] \exp \left(a \sqrt{\frac{s}{v}}\right) . }
\end{aligned}
$$

With the solution of the stream function, the fluid velocity components can be determined using Eq. (17).

The transient drag force exerted by the fluid on the spherical boundary $r=a^{+}$can be derived from a general expres- 
sion given by Happel and Brenner [20], with the result

$$
\begin{aligned}
F= & \eta \pi \int_{0}^{\pi} r^{3} \sin ^{3} \theta \frac{\partial}{\partial r}\left(\frac{E_{\mathrm{S}}^{2} \Psi}{r^{2} \sin ^{2} \theta}\right) r d \theta \\
& -\rho \pi \int_{0}^{\pi} r^{2} \sin ^{2} \theta \frac{\partial v_{\theta}}{\partial t} r d \theta .
\end{aligned}
$$

This force is equal to the rate of change of the particle momentum with respect to time,

$F=M \frac{d U}{d t}$,

where $M$ is the mass of the particle. After taking the Laplace transform of Eqs. (26) and (27) and using Eqs. (24), (25), and (17b), we obtain the general equation of motion of an electrophoretic sphere in response to the application of a step-function electric field,

$\bar{\mu}(s)=\beta^{2}\left(\frac{1}{a} \sqrt{\frac{v}{s}}+\frac{v}{a^{2} s}\right)\left[\frac{1}{s}+\sum_{n=1}^{\infty} \frac{C_{n}}{D_{n}^{2} v+s}-\bar{\mu}(s)\right]$,

where

$\beta=\sqrt{\frac{9 m}{2 M+m}}$,

and $m=(4 / 3) \pi a^{3} \rho$, which is the mass of the fluid having the same volume as the particle. Obviously, $0 \leqslant \beta \leqslant 3$, with the upper and lower bounds occurring at the limits $M / m=0$ and $M / m \rightarrow \infty$, respectively.

It is convenient to define a dimensionless time $T$ and its counterpart $S$ in the Laplace transform as

$T=t v \beta^{2} / a^{2}$,

$S=s a^{2} / \nu \beta^{2}$.

Rearranging Eq. (28) in terms of the new variables, we obtain

$\bar{\mu}^{*}(S)=\frac{1+\left(1-\beta^{2}\right) S+\beta S^{3 / 2}}{\left(S-\gamma_{1}\right)\left(S-\gamma_{2}\right)}\left(\frac{1}{S}+\sum_{n=1}^{\infty} \frac{C_{n}}{\omega_{n}+S}\right)$,

where

$\bar{\mu}^{*}(S)=\bar{\mu}(s) v \beta^{2} / a^{2}$,

$\omega_{n}=\left[\frac{(2 n-1) \pi a}{2 \beta \delta}\right]^{2}=\left[\frac{(2 n-1) \pi \kappa a}{2 \beta b}\right]^{2}$,

and $\gamma_{1}$ and $\gamma_{2}$ are the roots of the second-order equation

$S^{2}+\left(2-\beta^{2}\right) S+1=0$.

Equation (31) indicates that the dimensionless transient electrophoretic mobility $\mu$ is not only a function of the dimensionless time $T$ but also a function of the parameters $\kappa a / b$ $(=a / \delta)$ and $\beta$.

When $\beta \neq 2$, i.e., $\gamma_{1} \neq \gamma_{2}$, we take the inverse Laplace transform of Eq. (31) to result in

$$
\begin{aligned}
\mu= & -\frac{\beta}{\gamma_{1}-\gamma_{2}}\left[\sqrt{\gamma_{1}} \exp \left(\gamma_{1} T\right) \operatorname{erfc}\left(\sqrt{\gamma_{1} T}\right)\right. \\
& \left.-\sqrt{\gamma_{2}} \exp \left(\gamma_{2} T\right) \operatorname{erfc}\left(\sqrt{\gamma_{2} T}\right)\right] \\
+ & \sum_{n=1}^{\infty} C_{n}\left[\frac{\left(\gamma_{1}^{2}+\gamma_{1}\right) \operatorname{erfc}\left(\sqrt{\gamma_{1} T}\right)}{\left(\omega_{n}+\gamma_{1}\right)\left(\gamma_{2}-\gamma_{1}\right)} \exp \left(\gamma_{1} T\right)\right. \\
+ & \frac{\left(\gamma_{2}^{2}+\gamma_{2}\right) \operatorname{erfc}\left(\sqrt{\gamma_{2} T}\right)}{\left(\omega_{n}+\gamma_{2}\right)\left(\gamma_{1}-\gamma_{2}\right)} \exp \left(\gamma_{2} T\right) \\
+ & \frac{1+\omega_{n}\left(\gamma_{1}+\gamma_{2}+1\right)-i \beta \omega_{n}^{3 / 2} \operatorname{erf}\left(\sqrt{-\omega_{n} T}\right)}{\left(\omega_{n}+\gamma_{1}\right)\left(\omega_{n}+\gamma_{2}\right)} \\
& \left.\times \exp \left(-\omega_{n} T\right)\right],
\end{aligned}
$$

where $\operatorname{erf}(x)$ and $\operatorname{erfc}(x)$ represent the error function and complementary error function, respectively. Neutral buoyancy, where the particle and fluid densities are the same $(M / m=1)$, corresponds to $\beta=\sqrt{3}$. For the special case of $\beta=\sqrt{2}$ or the mass density ratio $M / m=7 / 4, \gamma_{1}=-\gamma_{2}=i$ and the above solution can be simplified to

$$
\begin{aligned}
\mu= & +\left[2 C_{\mathrm{F}}\left(\sqrt{\frac{2 T}{\pi}}\right)-1\right] \cos T \\
& +\left[2 S_{\mathrm{F}}\left(\sqrt{\frac{2 T}{\pi}}\right)-1\right] \sin T \\
& +\sum_{n=1}^{\infty} \frac{C_{n}}{1+\omega_{n}^{2}}\left[\left(1+\omega_{n}\right) \exp \left(-\omega_{n} T\right)\right. \\
& -\left(1+\omega_{n}\right) \cos T-\left(1-\omega_{n}\right) \sin T \\
& -i \sqrt{2} \omega_{n}^{3 / 2} \exp \left(-\omega_{n} T\right) \operatorname{erf}\left(\sqrt{-\omega_{n} T}\right) \\
& +2 C_{\mathrm{F}}\left(\sqrt{\frac{2 T}{\pi}}\right)\left(\cos T-\omega_{n} \sin T\right) \\
& \left.+2 S_{\mathrm{F}}\left(\sqrt{\frac{2 T}{\pi}}\right)\left(\sin T+\omega_{n} \cos T\right)\right]
\end{aligned}
$$

where $S_{\mathrm{F}}(s)$ and $C_{\mathrm{F}}(x)$ are the Fresnel sine and cosine integrals [21], respectively.

When $\beta=2$ or $M / m=5 / 8, \gamma_{1}=\gamma_{2}=1$ and the inverse transform of Eq. (31) yields

$$
\begin{aligned}
\mu= & +2 \sqrt{\frac{T}{\pi}}-(1+2 T) \exp (T) \operatorname{erfc}(\sqrt{T}) \\
& +\sum_{n=1}^{\infty} \frac{C_{n}}{\left(1+\omega_{n}\right)^{2}}\left\{\left[1+3 \omega_{n}+2 i \omega_{n}^{3 / 2} \operatorname{erf}\left(\sqrt{-\omega_{n} T}\right)\right]\right. \\
& \times \exp \left(-\omega_{n} T\right)+2\left(1+\omega_{n}\right) \sqrt{\frac{T}{\pi}} \\
& \left.-\left[1+3 \omega_{n}+2\left(1+\omega_{n}\right) T\right] \exp (T) \operatorname{erfc}(\sqrt{T})\right\} .
\end{aligned}
$$

The infinite series in Eqs. (35)-(37) are the contributions from the dynamic response of the electroosmotic flow at the particle surface after the electric field is suddenly applied. In 
the limit $\kappa a \rightarrow \infty$, these infinite series vanish and Eqs. (35)(37) reduce to the results obtained by Morrison [16]. The numerical calculations of Eqs. (35)-(37) for the normalized electrophoretic mobility $\mu$ as a function of the parameters $v t / a^{2}, \kappa a / b$, and $\beta$ will be presented in Section 5 .

\section{Transient electrophoresis of a sphere with a thick double layer}

In this section, we first consider the transient translation of a spherical particle of radius $a$ caused by a suddenly applied body force, and then extend its result to the case of electrophoretic motion of a dielectric sphere with a thick electric double layer. At the time $t=0$, a constant force $F_{\mathrm{A}}$ is imposed on the particle in the positive $z$ direction and maintained afterwards. When the Reynolds number is small, the ambient fluid motion is governed by Eq. (16). The initial and boundary conditions for the flow field are still given by Eqs. (19)-(21), but with Eq. (20b) replaced by

$r=a: \quad v_{\theta}=-U \sin \theta$,

where $U(t)$ is the time-dependent translational velocity of the particle to be determined, which can be expressed according to Stokes law as $U=U_{\infty} \mu(t)$ with

$U_{\infty} \equiv \frac{F_{\mathrm{A}}}{6 \pi \eta a}$.

Again, $\mu(0)=0$ and $\mu(\infty)=1$. Taking the Laplace transform of Eq. (16) and applying conditions (19), (20a), (21), and (38) again yields a solution for the stream function in the form of Eq. (24), but here the coefficients $A$ and $B$ become

$A=-\left(1+a \sqrt{\frac{s}{v}}+\frac{a^{2}}{3} \frac{s}{v}\right) \bar{\mu}(s)$,

$B=\bar{\mu}(s) \exp \left(a \sqrt{\frac{s}{v}}\right)$.

The transient drag force $F$ acting on the spherical boundary $r=a$ by the fluid can also be determined from Eq. (26). The sum of this hydrodynamic force and the applied force on the particle is equal to the rate of change of the particle momentum,

$F_{\mathrm{A}}+F=M \frac{d U}{d t}$.

After taking the Laplace transform of Eqs. (26) and (41) and using Eqs. (17b), (24), and (40), one obtains

$\bar{\mu}(s)=\frac{1}{s}\left(\frac{a^{2} s}{\beta^{2} v}+1+a \sqrt{\frac{s}{v}}\right)^{-1}$.

In terms of the dimensionless variables defined by Eqs. (30) and (32), Eq. (42) becomes

$\bar{\mu}^{*}(S)=\frac{1+S-\beta S^{1 / 2}}{S\left(S-\gamma_{1}\right)\left(S-\gamma_{2}\right)}$, where $\gamma_{1}$ and $\gamma_{2}$ are the roots of Eq. (34).

When $\beta \neq 2$, i.e., $\gamma_{1} \neq \gamma_{2}$, the inverse Laplace transform of Eq. (43) leads to

$$
\begin{aligned}
\mu=1-\frac{\beta}{\gamma_{1}-\gamma_{2}}[ & \sqrt{\gamma_{1}} \exp \left(\gamma_{2} T\right) \operatorname{erfc}\left(\sqrt{\gamma_{2} T}\right) \\
& \left.-\sqrt{\gamma_{2}} \exp \left(\gamma_{1} T\right) \operatorname{erfc}\left(\sqrt{\gamma_{1} T}\right)\right] .
\end{aligned}
$$

For the special case of $\beta=\sqrt{2}, \gamma_{1}=-\gamma_{2}=i$ and the above formula reduces to

$$
\begin{aligned}
\mu=1 & +\left[2 S_{\mathrm{F}}\left(\sqrt{\frac{2 T}{\pi}}\right)-1\right] \cos T \\
& -\left[2 C_{\mathrm{F}}\left(\sqrt{\frac{2 T}{\pi}}\right)-1\right] \sin T .
\end{aligned}
$$

When $\beta=2, \gamma_{1}=\gamma_{2}=1$ and the inverse transform of Eq. (43) gives

$\mu=1-2 \sqrt{\frac{T}{\pi}}+(2 T-1) \exp (T) \operatorname{erfc}(\sqrt{T})$.

We now consider the transient electrophoresis of a small charged sphere surrounded by a thick electric double layer in an electrolyte solution, i.e., with $\kappa a \rightarrow 0$. At the time $t=0$, a step function electric field with constant strength $E_{\infty}$ is imposed. Because the gradient of the electrostatic potential inside the double layer is characterized by the quantity $\kappa|\zeta|$, which is small, the effect of the electric force in the equation of fluid motion is negligible. Thus, in the limit of small Reynolds number, the flow field around the electrophoretic particle is also governed by Eqs. (16), (19), (20a), (21), and (38). The Debye-Hückel approximation for a dielectric sphere of radius $a$ leads to a formula for the charge on the particle surface as $q=a \varepsilon \zeta$. The applied electric field exerts a body force on the charged particle with the magnitude

$F_{\mathrm{A}}=q E_{\infty}=a \varepsilon \zeta E_{\infty}$.

Therefore, the transient response of the particle migration to the imposed step function electric field is the same as that presented above for the body-force-driven motion with the suddenly applied force given by the above equation or, according to Hückel equation [2], with the steady particle velocity $U_{\infty}$ in Eq. (39) replaced by

$U_{\infty} \equiv \frac{\varepsilon \zeta E_{\infty}}{6 \pi \eta}$.

The result for the time-dependent electrophoretic mobility of the charged particle for various values of the parameter $\beta$ is again given by Eqs. (44)-(46) and will be discussed in Section 5 .

\section{Results and discussion}

When a spherical dielectric particle of radius $a$ surrounded by a thin electric double layer in an electrolyte 


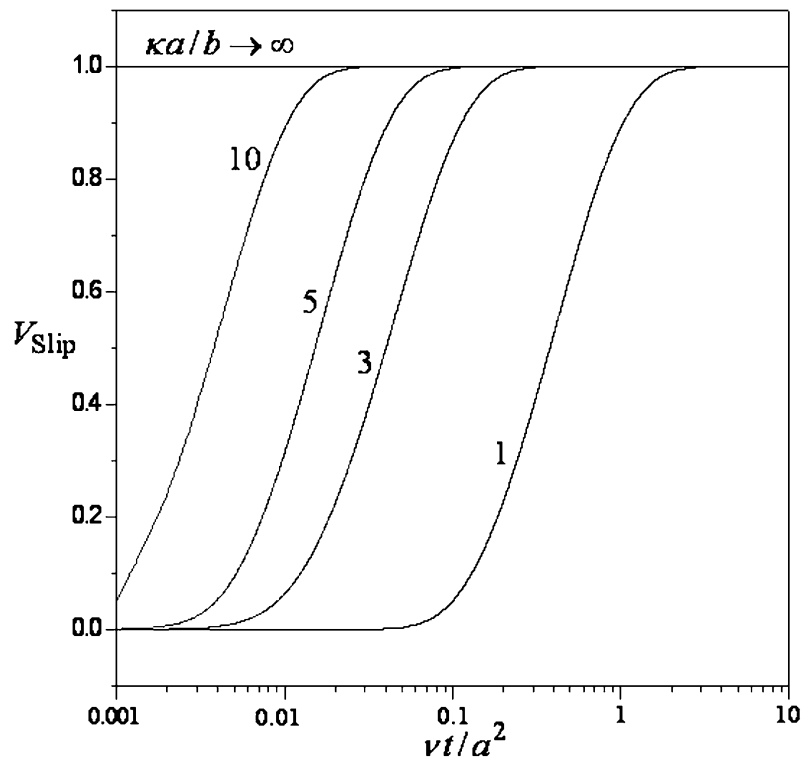

(a)

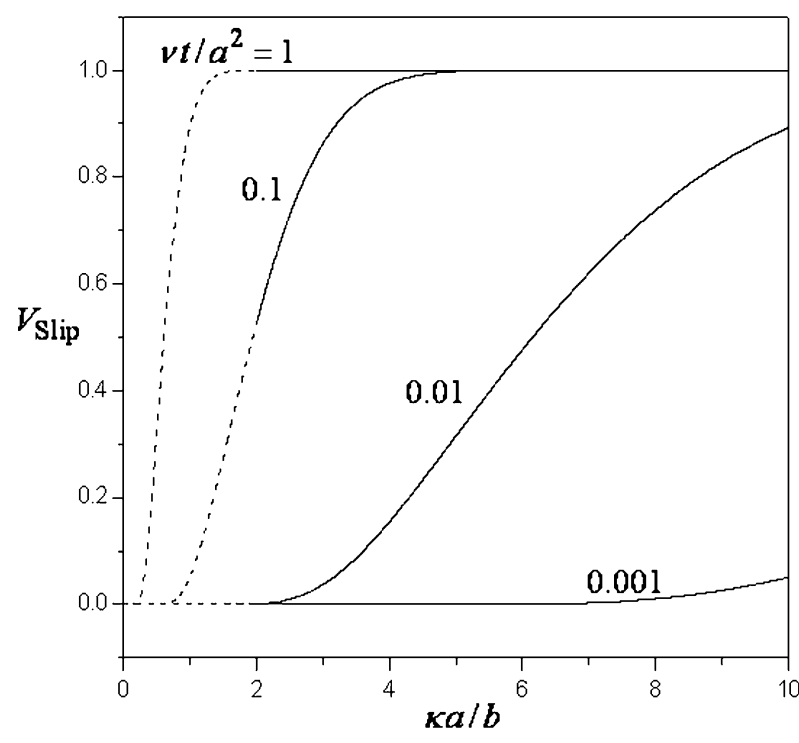

(b)

Fig. 1. Plots of the normalized slip velocity $V_{\text {slip }}$ at the particle surface (a) versus the dimensionless time $v t / a^{2} ;(\mathrm{b})$ versus the parameter $\kappa a / b$.

solution is subjected to an applied electric field of constant strength $E_{\infty}$ from time $t=0$, the apparent slip velocity of the fluid at the particle surface (or more precisely, at the outer boundary of the double layer) caused by the electroosmotic effect, which drives the electrophoretic motion of the particle, is given by the last term of Eq. (20b). A normalized quantity of this time-dependent slip velocity can be expressed as $V_{\text {slip }}=1+\sum_{n=1}^{\infty} C_{n} \exp \left(-D_{n}^{2} v t\right)$, which is a function of the parameter $v t / \delta^{2}\left[=(\kappa a / b)^{2}\left(v t / a^{2}\right)\right]$. Fig. 1 shows the results of $V_{\text {slip }}$ for various values of $\kappa a / b$ and $v t / a^{2}$. The case of $\kappa a / b<3$, which is hardly consistent with the assumption of a thin double layer at the particle surface, is considered here for the sake of numerical comparison. It can be seen that the apparent slip velocity at the particle surface increases monotonically and rapidly with the time from zero at $t=0$ to its steady-state magnitude as $t \rightarrow \infty$, as expected, for any given finite value of $\kappa a / b$. For a fixed value of $v t / a^{2}$, the normalized slip velocity decreases monotonically with a decrease in $\kappa a / b$ or an increase in the thickness of the double layer. Owing to the fact that the response time of the fluid within an infinitely thin double layer to the applied electric field is zero, $V_{\text {slip }}$ equals unity in the limit of $\kappa a \rightarrow \infty$ irrespectively of the duration. For all cases satisfying the thin double layer assumption $(\kappa a / b \geqslant 3)$, the slip velocity approaches its steady quantity rapidly, indicating that it is justified to neglect the relaxation effect of this slip velocity in Morrison [16] analysis.

The expressions for the time-dependent electrophoretic mobility of a dielectric sphere in response to the application of a step-function electric field normalized by its corresponding steady-state quantity, $\mu$, are given by Eqs. (35)(37) for the case of relatively large values of $\kappa a$ and by Eqs. (44)-(46) for the case of $\kappa a \rightarrow 0$. In Fig. 2, this nor- malized particle mobility is plotted versus the dimensionless time $v t / a^{2}$ and the mass density parameter $\beta$ for three values of $\kappa a / b$. As expected, the electrophoretic mobility of the particle increases monotonically (but not as rapidly as the apparent slip velocity at the particle surface shown in Fig. 1a does) with the time from zero at $t=0$ to its steadystate magnitude as $t \rightarrow \infty$ for any specified value of $\kappa a / b$ and finite value of $\beta$. For constant values of $\kappa a / b$ and $v t / a^{2}$, $\mu$ is a monotonic increasing function of $\beta$, meaning that a heavier particle lags behind a lighter one in the development of the electrophoretic mobility. In the limiting case of $\beta=0$ or $M / m \rightarrow \infty$, the particle mobility vanishes regardless of the values of $\kappa a$ and $t$.

The normalized electrophoretic velocity of a dielectric sphere as a function of the dimensionless time is also plotted with $\kappa a / b$ as a parameter in Fig. 3 for the cases of $\beta^{2}=1$ (or $M / m=4)$ and $\beta^{2}=6$ (or $M / m=1 / 4$ ). When the electric double layer surrounding the particle is relatively thin, the particle mobility decreases monotonically with a decrease in $\kappa a / b$ for fixed values of $\beta$ and $v t / a^{2}$. This behavior is expectable knowing that the apparent slip velocity caused by the electroosmotic flow at the particle surface decreases with a decrease in the value of $\kappa a / b$ indicated by Fig. 1 . When the double layer of the particle is thick, the normalized electrophoretic velocity can have magnitudes comparable to those for a particle with a thin double layer (and is still smaller than that for a particle with $\kappa a \rightarrow \infty$ ) in the initial stage, but will be much slower as the dimensionless time $v t / a^{2}$ becomes greater than about unity, regardless of the value of $\beta$.

Fig. 4 shows plots of the dimensionless acceleration $\left(a^{2} / v\right) d \mu / d t$ of a spherical particle undergoing transient electrophoresis versus the dimensionless time $v t / a^{2}$ with $\beta$ 


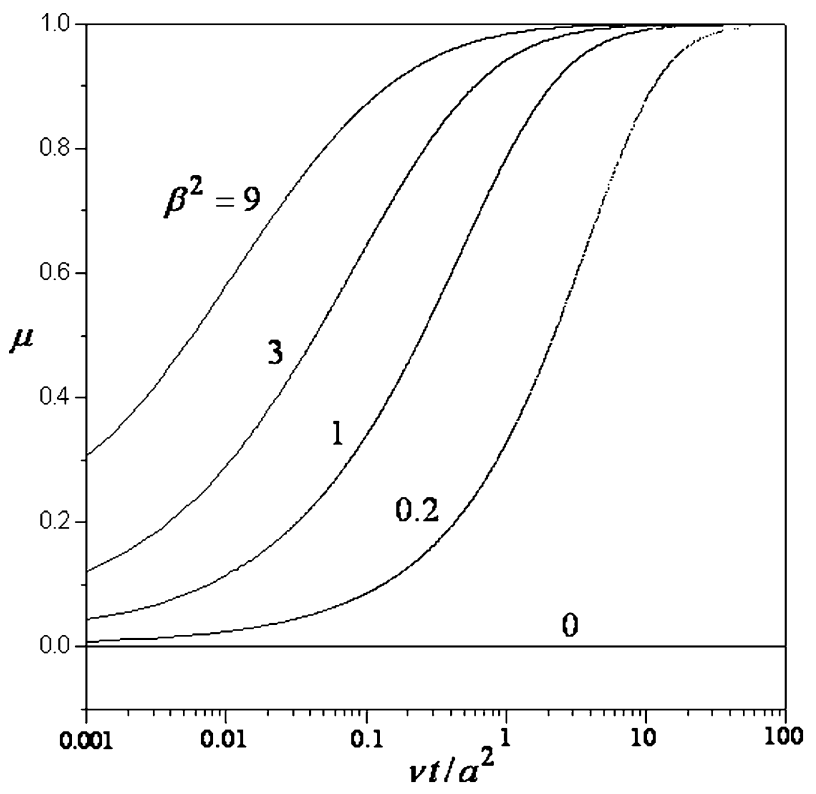

(a)

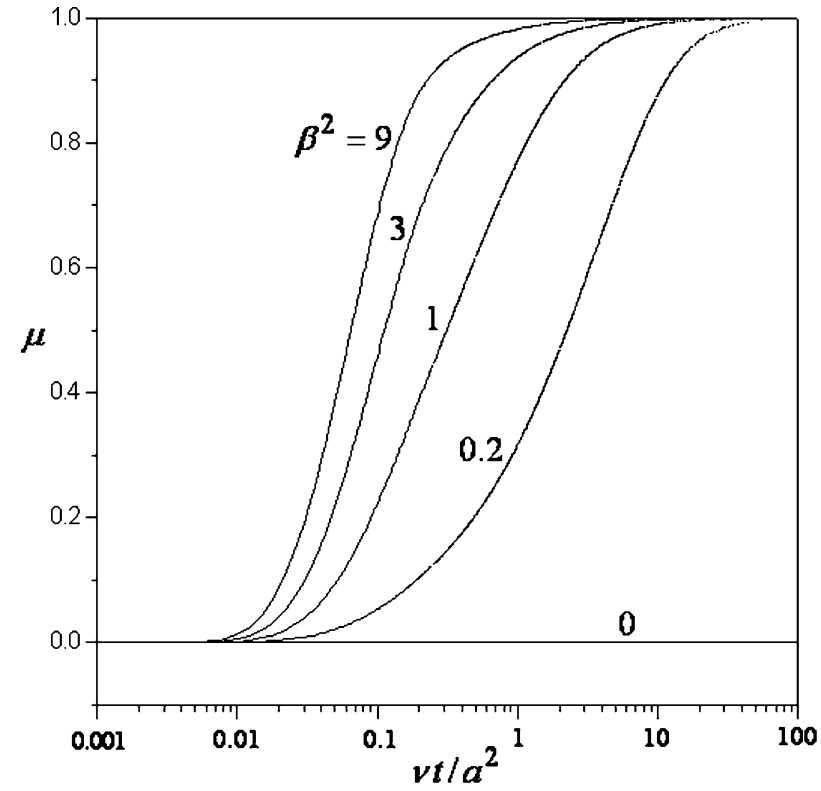

(b)

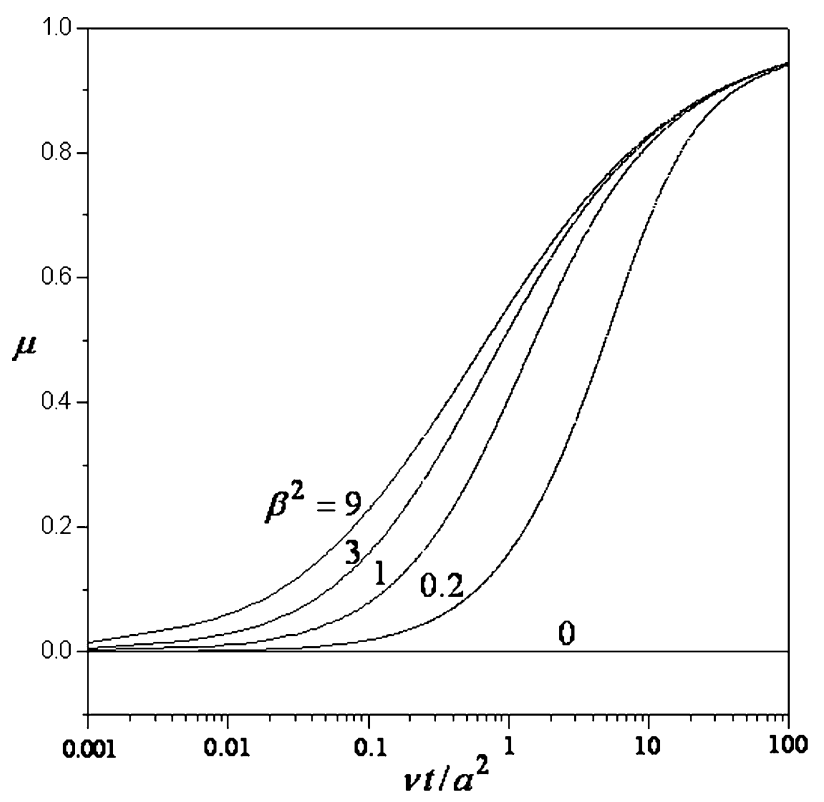

(c)

Fig. 2. Plots of the normalized electrophoretic mobility $\mu$ versus the dimensionless time $v t / a^{2}$ with $\beta^{2}$ as a parameter: (a) $\kappa a / b \rightarrow \infty$; (b) $\kappa a / b=3$; (c) $\kappa a / b=0$.

and $\kappa a / b$ as parameters. For the limiting case of $\kappa a \rightarrow \infty$ illustrated in Fig. 4a, the acceleration is a monotonic decreasing function of the time and is infinite at the instant the electric field is imposed. The singular result of an infinite initial acceleration is due to the finite slip velocity at the particle surface, which drives the electrophoretic movement of the particle, even at the time $t=0$ as shown in Fig. 1a. For the case of a sphere with a thin but finite double layer as depicted in Fig. 4b, the dimensionless acceleration first increases with the time from zero at $t=0$ to a maximum at a small finite value of $v t / a^{2}$, and then decreases with the time monotonically. The result of a vanishing initial acceleration in this case is due to the zero slip velocity at the particle surface at $t=0$ as indicted in Fig. 1a. For the limiting case of $\kappa a=0$ exhibited in Fig. $4 c$, the acceleration is a monotonic decreasing function of the time but is finite at the instant the electric field is applied. For any given values of $\kappa a / b$ and $\beta$, as expected, the acceleration of the electrophoretic particle decreases rapidly after $v t / a^{2}$ equal to about 0.1 and vanishes in the limit $v t / a^{2} \rightarrow \infty$.

As a typical example, we now consider the situation of a neutrally buoyant particle (with $\beta^{2}=3$ or $M / m=1$ ) shown in Fig. 2. The instantaneous electrophoretic mobility of the particle reaches $95 \%$ of its terminal value in the dimension- 


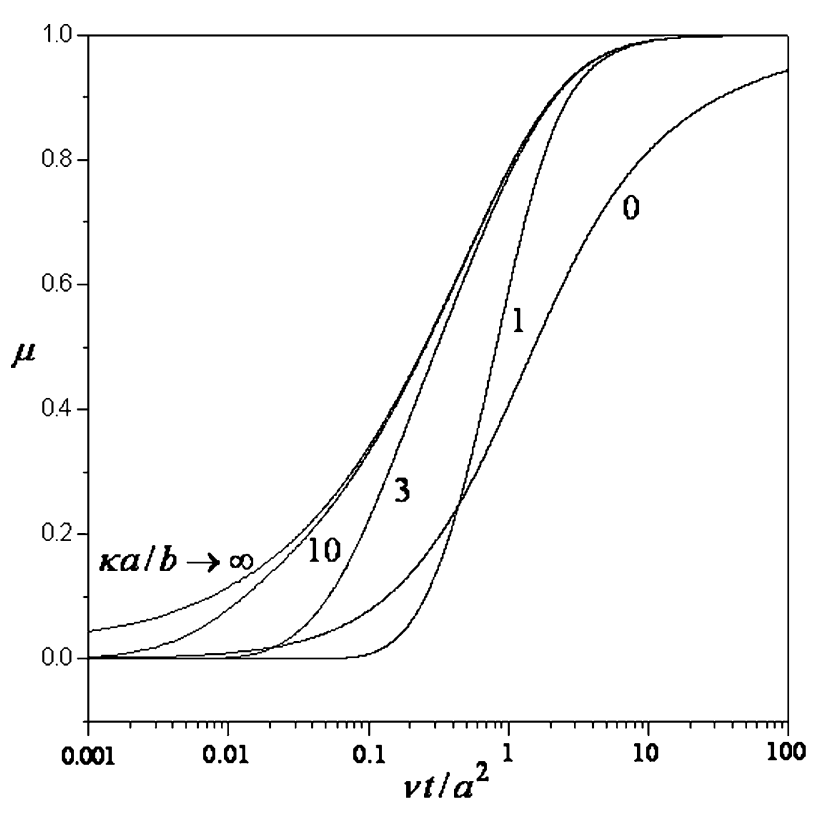

(a)

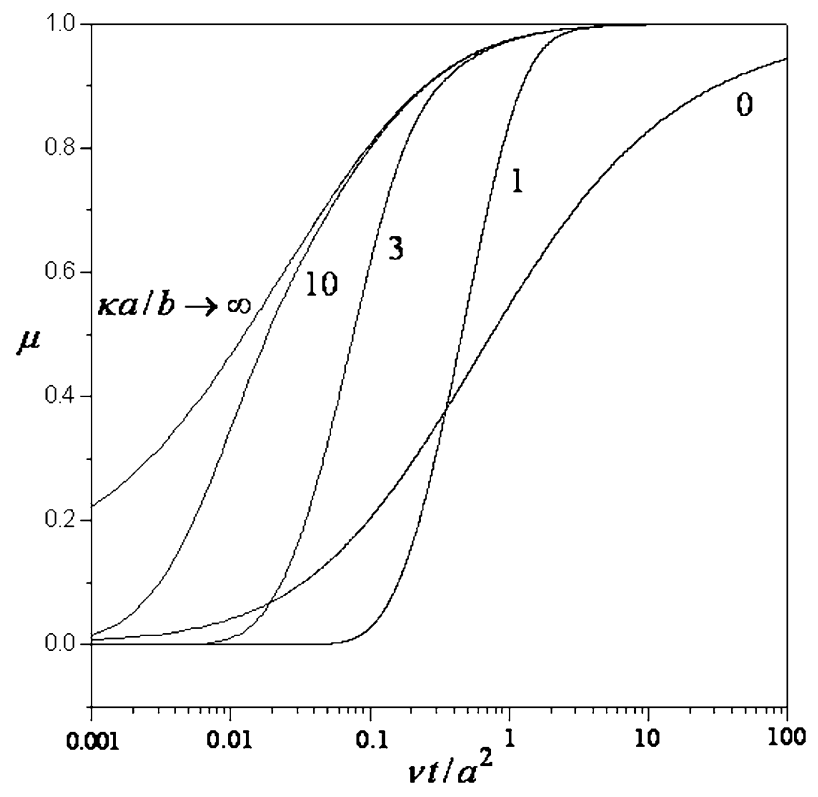

(b)

Fig. 3. Plots of the normalized electrophoretic mobility $\mu$ versus the dimensionless time $v t / a^{2}$ with $\kappa a / b$ as a parameter: (a) $\beta^{2}=1$; (b) $\beta^{2}=6$.

less time $v t / a^{2}$ equal to about 1.2 and 127 for the cases of a thin double layer and of a thick double layer, respectively, independent of the applied electric field and the zeta potential. When the particle is suspended in an aqueous solution (with $v$ of the order $10^{-6} \mathrm{~m}^{2} / \mathrm{s}$ ) and has a radius of the order $1 \mu \mathrm{m}$ (for the case of thin double layer) or $0.1 \mu \mathrm{m}$ (for the case of thick double layer), these relaxation responses correspond to times of order $\mu$ s. For a particle with a smaller value of $\beta$ or a greater value of $M / m$, the relaxation time for transient electrophoresis can be an order of magnitude longer, but this response time is still negligible in practical applications. Consequently, the electrophoretic velocity of a dielectric sphere will closely follow the Smoluchowski equation (for $\kappa a \gg 1$ ) or Hückel equation (for $\kappa a \ll 1$ ) with the instantaneous applied electric field. The diffusion time scale for an electrolyte having a typical diffusivity of the order $10^{-9} \mathrm{~m}^{2} / \mathrm{s}$ across a thin double layer of $10 \mathrm{~nm}$ thickness is about $0.1 \mu$ s. This time scale is an order of magnitude smaller than the viscous relaxation time, which justifies the assumption of equilibrium ion distributions inside the thin double layer. Although the dimensionless transition times shown in Figs. 1-4 are down to the order $10^{-3}$, it is understood that these thin double layer results may be accurate only for the case of large particles.

\section{Conclusions}

An analysis of the transient electrophoresis of a dielectric sphere suspended in an electrolyte solution in response to a step change in the applied electric field, which will approximate the fields encountered in most technological applications, is presented in this work. When the electrical double layer surrounding the particle is thin compared with the particle radius, the apparent slip velocity at the particle surface is derived from the dynamic electroosmotic velocity of the fluid at the outer boundary of the double layer adjacent to a plane wall. By solving the unsteady momentum conservation equation applicable to the systems of a particle with a thin but finite double layer and of a particle with a thick double layer, closed-form expressions for the dynamic response of the flow field of the suspending fluid and the electrophoretic mobility of the particle as functions of relevant parameters are obtained. These results show that, when the double layer surrounding the particle is relatively thin, the particle mobility decreases monotonically with a decrease in the electrokinetic particle radius $\kappa a$ for fixed values of the mass density parameter $\beta$ and the dimensionless time $v t / a^{2}$. When the double layer of the particle is thick, the electrophoretic mobility can have magnitudes comparable to those for a particle with a thin double layer in the initial stage, but will be much smaller as $v t / a^{2}$ becomes greater. For the case of an electrophoretic sphere with a thin but finite double layer, the acceleration of the particle first increases with the time from zero at $t=0$ to a maximum at a small value of $v t / a^{2}$, and then decreases with the time and vanishes in the limit $v t / a^{2} \rightarrow \infty$.

Our results, which provide useful insight into the actual phenomena regarding the transient response of a charged particle to a sudden application of a constant electric field, show that the effect of the relaxation time for transient electrophoresis in general is negligible, irrespective of the applied electric field, the zeta potential, and the value of $\kappa a$. Also, these results will serve as useful limiting solution against which the results of more sophisticated models (which account for the polarization effect of the mobile ions 


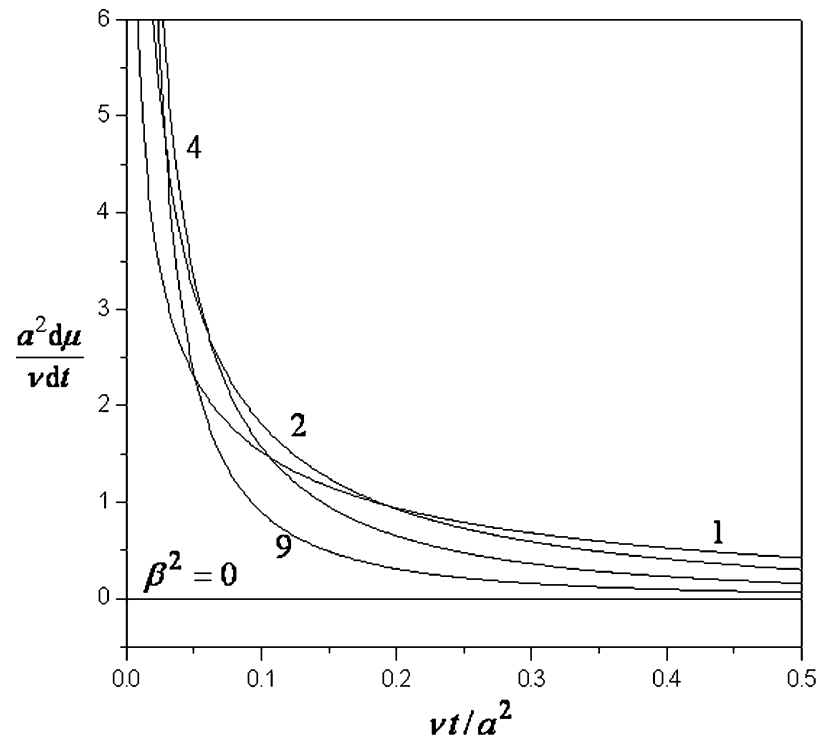

(a)

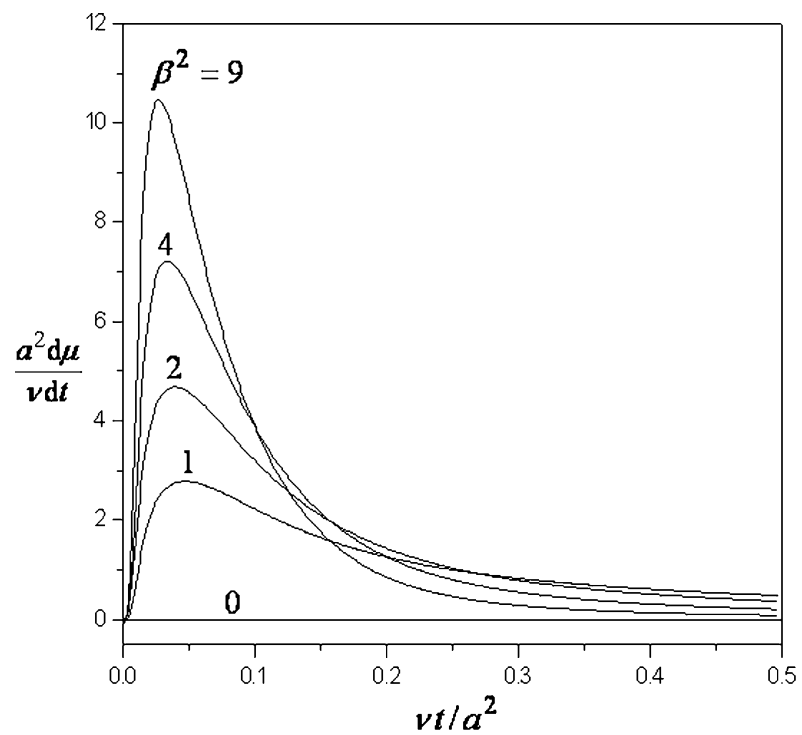

(b)

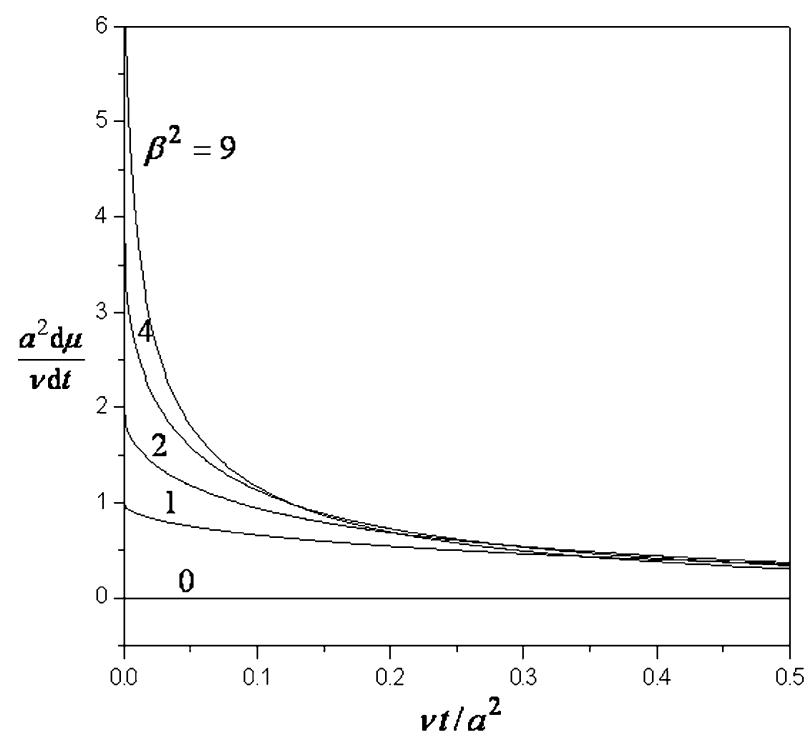

(c)

Fig. 4. Plots of the normalized electrophoretic acceleration $\left(a^{2} / v\right) d \mu / d t$ versus the dimensionless time $v t / a^{2}$ with $\beta^{2}$ as a parameter: (a) $\kappa a / b \rightarrow \infty$; (b) $\kappa a / b=3$; (c) $\kappa a / b=0$.

in the thin double layer [11,12], for instance, or even deal with a particle with an arbitrary value of $\kappa a$ ) may be benchmarked.

\section{Acknowledgment}

This research was partially supported by the National Science Council of the Republic of China.

\section{References}

[1] M. Smoluchowski, Bull. Acad. Sci. Cracovie (1903) 182.

[2] E. Hückel, Phys. Z. 25 (1924) 204.
[3] D.C. Henry, Proc. Roy. Soc. London Ser. A 133 (1931) 106-129.

[4] F. Booth, Proc. Roy. Soc. London Ser. A 203 (1950) 514-533.

[5] P.H. Wiersema, A.L. Loeb, J.Th.G. Overbeek, J. Colloid Interface Sci. 22 (1966) 78-99.

[6] F.A. Morrison, J. Colloid Interface Sci. 34 (1970) 210-214.

[7] S.S. Dukhin, B.V. Derjaguin, in: E. Matijevic (Ed.), Surface and Colloid Science, vol. 7, Wiley, New York, 1974.

[8] R.W. O’Brien, L.R. White, J. Chem. Soc. Faraday Trans. 2 (74) (1978) $1607-1626$.

[9] R.J. Hunter, Zeta Potential in Colloid Science, Academic Press, London, 1981.

[10] H. Ohshima, T.W. Healy, L.R. White, J. Chem. Soc. Faraday Trans. 2 (79) (1983) 1613-1628.

[11] R.W. O’Brien, J. Colloid Interface Sci. 92 (1983) 204-216.

[12] S.B. Chen, H.J. Keh, J. Fluid Mech. 238 (1992) 251-276.

[13] J.H. Masliyah, Electrokinetic Transport Phenomena, AOSTRA, Edmonton, 1994. 
[14] R.W. O’Brien, D.W. Cannon, W.N. Rowlands, J. Colloid Interface Sci. 173 (1995) 406-418.

[15] M. Rasmusson, B. Akerman, Langmuir 14 (1998) 3512-3516.

[16] F.A. Morrison, J. Colloid Interface Sci. 29 (1969) 687-691.

[17] F.A. Morrison, J. Colloid Interface Sci. 36 (1971) 139-145.

[18] C.F. Ivory, J. Colloid Interface Sci. 100 (1984) 239-249.
[19] H.J. Keh, H.C. Tseng, J. Colloid Interface Sci. 242 (2001) 450459.

[20] J. Happel, H. Brenner, Low Reynolds Number Hydrodynamics, Martinus Nijhoff, Dordrecht, 1983.

[21] M.R. Spiegel, Mathematical Handbook, McGraw-Hill, New York, 1981. 